\title{
Petroleum Reservoir Engineering by Non-linear Singular Integral Equations
}

\author{
E. G. Ladopoulos \\ Interpaper Research Organization \\ 8, Dimaki Str., Athens, GR - 106 72, Greece
}

$\begin{array}{lc}\text { Received: August 28, } 2011 & \text { Accepted: September 12, } 2011 \quad \text { Published: December 31, } 2011 \\ \text { doi:10.5539/mer.v1n1p2 } & \text { URL: http://dx.doi.org/10.5539/mer.v1n1p2 }\end{array}$

\begin{abstract}
For the determination of the properties of several reservoir materials, when oil reserves are moving through porous media, a new mathematical approach is proposed. Such problem is very much important for petroleum reservoir engineering. Thus, the above mentioned problem is reduced to the solution of a non-linear singular integral equation, which is numerically evaluated by using the Singular Integral Operators Method (S.I.O.M.). Beyond the above, several properties are analyzed and investigated for the porous medium equation, defined as a Helmholtz differential equation.

Finally, an application is given for a well testing to be checked when an heterogeneous oil reservoir is moving in a porous medium. Hence, by using the S.I.O.M., then the pressure response from the well test conducted in the above heterogeneous oil reservoir, is numerically calculated and investigated.
\end{abstract}

Keywords: Singular Integral Operators Method (S.I.O.M.), Non-linear singular integral equation, Oil reserves, Porous media, Petroleum reservoir engineering, Helmholtz differential equation

\section{Introduction}

The study of the movement of oil reserves through porous media is very much important problem on petroleum reservoir engineering. Therefore, by applying a well test analysis, then a history matching process takes place for the determination of the properties of the reservoir materials. The movement of oil reserves through porous media, produces both single-phase and multiphase flows. Furthermore, if a well test is conducted, then the well is subjected to a change of the flow rate and the pressure response can be further measured. For the determination of several petroleum reservoir parameters, such as permeability, then numerical calculations should be used, as analytical solutions in most cases are not possible to be derived.

During the past years several variants of the Boundary Element Method were used for the solution of petroleum reservoir engineering problems. At the end of eight's Lafe and Cheng (1987) proposed a BEM for the solution of steady flows in heterogeneous solids. During the same period Masukawa and Horne (1988) and Numbere and Tiab (1988) applied boundary elements for steady state problems of streamline tracking.

Furthermore, Kikani and Horne (1992) solved transient problems by using a Laplace space boundary element model, for the analysis of well tests in several arbitrarily shaped reservoirs. Beyond the above, Koh and Tiab (1993) used boundary elements to describe the flow around tortuous horizontal wells, for homogeneous, or piecewise homogeneous reservoirs.

Sato and Horne (1993, 306-314; 1993, 315-322) applied perturbation boundary elements for the study of heterogeneous reservoirs. Also, El Harrouni, Quazar, Wrobel and Cheng (1996) proposed the use of a transformed form of Darcy's law combined with dual reciprocity boundary element method to handle heterogeneity. On the other hand, Onyejekwe (1997) applied a Green element method to isothermal flows with second order reactions. The same author (Onyejekwe O.O., 1998, 293-312; Onyejekwe O.O., 1998, 313-330) used a combined method of boundary elements together with finite elements for the study of heterogeneous reservoirs. Beyond the above, Taigbenu and Onyejekwe (1997) applied a transient one-dimensional transport equation by using a mixed Green element method.

During the last years several non-linear singular integral equation methods were used successfully by Ladopoulos (1991) - (2000, Springer Verlag) for the solution of applied problems of solid mechanics, 
elastodynamics, structural analysis, fluid mechanics and aerodynamics. Thus, in the present research, the non-linear singular integral equations will be used in order to determine the properties of the reservoir materials, when oil reserves are moving through porous solids.

By using therefore, the Singular Integral Operators Method (S.I.O.M.), then the pressure response from the well test conducted in an heterogeneous reservoir will be computed. Also, some properties of the porous medium equation, which is a Helmholtz differential equation, are proposed and investigated. Thus, basic properties of the fundamental solution will be analyzed and investigated.

Finally, an application is given for a well testing to be investigated when an heterogeneous oil reservoir is moving in a porous medium. Then this problem will be solved by using the Singular Integral Operators Method and so the pressure response from the well test conducted in this heterogeneous oil reservoir, will be computed.

Hence, the non-linear singular integral equation methods which were used with big success for the solution of several engineering problems of fluid mechanics, hydraulics, aerodynamics, solid mechanics, elastodynamics, and structural analysis, are further extended in the present study for the solution of oil reservoir engineering problems. In such a case the non-linear singular integral equations are used for the solution of one of the most important and interesting problems for petroleum engineers.

\section{Well Test Analysis for Oil Reservoir}

Oil well test analysis is a kind of an important history matching process for the determination of the properties of reservoir materials. Thus, during the movement of oil reservoir through porous media, then both single-phase and multiphase flow occurs. Also, when a petroleum well test is conducted then the well is subjected to a change of its flow rate and the resulting pressure response is possible to be measured. Moreover, this pressure is compared to analytical or numerical models in order to estimate reservoir parameters such as permeability.

In general an oil reservoir well test in a single-phase reservoir is calculated by using the porous medium equation:

$$
\nabla \bullet\left(\frac{\lambda}{\phi \xi} \nabla p\right)=c_{t} \frac{\partial p}{\partial t}
$$

in which $\lambda$ denotes the permeability, $\phi$ the porosity, $\xi$ the viscosity, $p$ the pressure of the reservoir, $t$ the time and $c_{t}$ the compressibility.

By replacing variables as follows:

$$
u=\left(\frac{\lambda}{\phi \xi}\right)^{1 / 2} p
$$

then (2.1) can be written as:

$$
\nabla^{2} u+\lambda^{\prime} u=0
$$

with :

$$
\lambda^{\prime}=\frac{\nabla^{2}(\lambda / \phi \xi)^{1 / 2}}{(\lambda / \phi \xi)^{1 / 2}}
$$

Hence, eq. (2.3) is a Helmholtz differential equation.

Beyond the above, consider by $u^{*}(\mathbf{x}, \mathbf{y})$ the fundamental solution of any point $\mathbf{y}$, because of the source point $\mathbf{x}$. Then the fundamental solution can be given by the following equation:

$$
\nabla^{2} u^{*}(\mathbf{x}, \mathbf{y})+\lambda^{\prime} u^{*}(\mathbf{x}, \mathbf{y})+\delta(\mathbf{x}, \mathbf{y})=0
$$

which may be further written as:

$$
u_{, i i}^{*}(\mathbf{x}, \mathbf{y})+\lambda^{\prime} u^{*}(\mathbf{x}, \mathbf{y})+\delta(\mathbf{x}, \mathbf{y})=0
$$

Thus, eq. (2.5) is the Helmholtz potential equation governing the fundamental solution.

Consider further by $u^{*}$ the fundamental solution chosen so that to enforce the Helmholtz equation in terms of the function $u$, in a weak form. Then the weak form of Helmholtz equation will be written as following: 
in the solution domain $\Omega$.

$$
\int_{\Omega}\left(\nabla^{2} u+\lambda^{\prime} u\right) u^{*} d \Omega=0
$$

Also, by applying the divergence theorem once in (2.6), one obtains a symmetric weak form:

$$
\int_{\partial \Omega} n_{i} u_{, i} u^{*} d S-\int_{\Omega} u_{, i} u_{, i}^{*} d \Omega+\int_{\Omega} \lambda^{\prime} u u^{*} d \Omega=0
$$

in which $\mathbf{n}$ denotes the outward normal vector of the surface $S$.

Therefore, in the symmetric weak form the function $u$ and the fundamental solution $u^{*}$ are only required to be first - order differentiable. By applying further the divergence theorem twice in (2.6) we have:

$$
\int_{\partial \Omega} n_{i} u_{, i} u^{*} d S-\int_{\partial \Omega} n_{i} u u_{, i}^{*} d S+\int_{\Omega} u\left(u_{, i i}^{*}+\lambda^{\prime} u^{*}\right) d \Omega=0
$$

Hence, (2.8) is the asymmetric weak form and the fundamental solution $u^{*}$ is required to be second - order differentiable. On the other hand, $u$ is not required to be differentiable in the domain $\Omega$.

By combining eqs (2.5) and (2.8), then one obtains:

$$
u(\mathbf{x})=\int_{\partial \Omega} n_{i}(\mathbf{y}) u_{, i}(\mathbf{y}) u^{*}(\mathbf{x}, \mathbf{y}) d S-\int_{\partial \Omega} n_{i}(\mathbf{y}) u(\mathbf{y}) u_{, i}^{*}(\mathbf{x} ., \mathbf{y}) d S
$$

which can be further written as:

$$
u(\mathbf{x})=\int_{\partial \Omega} q(\mathbf{y}) u^{*}(\mathbf{x}, \mathbf{y}) d S-\int_{\partial \Omega} u(\mathbf{y}) R^{*}(\mathbf{x}, \mathbf{y}) d S
$$

where $q(\mathbf{y})$ denotes the potential gradient along the outward normal direction of the boundary surface:

$$
q(\mathbf{y})=\frac{\partial u(\mathbf{y})}{\partial n_{y}}=n_{k}(\mathbf{y}) u_{, k}(\mathbf{y}), \quad \mathbf{y} \in \partial \Omega
$$

and the kernel function:

$$
R^{*}(\mathbf{x}, \mathbf{y})=\frac{\partial u^{*}(\mathbf{x}, \mathbf{y})}{\partial n_{y}}=n_{k}(\mathbf{y}) u_{, k}^{*}(\mathbf{x}, \mathbf{y}), \mathbf{y} \in \partial \Omega
$$

By differentiating (2.10) with respect to $x_{k}$, we obtain the integral equation for potential gradients $u_{k}(\mathbf{x})$ by the following formula:

$$
\frac{\partial u(\mathbf{x})}{\partial x_{k}}=\int_{\partial \Omega} q(\mathbf{y}) \frac{\partial u^{*}(\mathbf{x}, \mathbf{y})}{\partial x_{k}} d S-\int_{\partial \Omega} u(\mathbf{y}) \frac{\partial R^{*}(\mathbf{x}, \mathbf{y})}{\partial x_{k}} d S
$$

\section{Fundamental Solution's Basic Properties}

Beyond the above, we rewrite the weak form of (2.5) governing the fundamental solution, as follows:

$$
\int_{\Omega}\left[\nabla^{2} u^{*}(\mathbf{x}, \mathbf{y})+\lambda^{\prime} u^{*}(\mathbf{x}, \mathbf{y})\right] c d \Omega+c=0, \quad \mathbf{x} \in \Omega
$$

where $\mathrm{c}$ denotes a constant, considering as the test function.

Also, eq. (3.1) can be written as:

$$
\int_{\Omega}\left[u_{, i i}^{*}(\mathbf{x}, \mathbf{y})+\lambda^{\prime} u^{*}(\mathbf{x}, \mathbf{y})\right] d \Omega+1=0, \quad \mathbf{x} \in \Omega
$$

Furthermore, (3.2) takes the form:

$$
\int_{\partial \Omega} n_{i}(\mathbf{y}) u_{, i}^{*}(\mathbf{x}, \mathbf{y}) d S+\int_{\Omega} \lambda^{\prime} u^{*}(\mathbf{x}, \mathbf{y}) d \Omega+1=0, \quad \mathbf{x} \in \Omega
$$

By considering further an arbitrary function $u(\mathbf{x})$ in $\Omega$ as the test function, then the weak form of (2.5) will be written as: 


$$
\int_{\Omega}\left[\nabla^{2} u^{*}(\mathbf{x}, \mathbf{y})+\lambda^{\prime} u^{*}(\mathbf{x}, \mathbf{y})+\delta(\mathbf{x}, \mathbf{y})\right] u(\mathbf{x}) d \Omega=0, \quad \mathbf{x} \in \Omega
$$

and also as:

$$
\int_{\Omega}\left[u_{, i i}^{*}(\mathbf{x}, \mathbf{y})+\lambda^{\prime} u^{*}(\mathbf{x}, \mathbf{y})\right] u(\mathbf{x}) d \Omega+u(\mathbf{x})=0, \quad \mathbf{x} \in \Omega
$$

Finally, (3.5) takes the form:

$$
\int_{\partial \Omega} \Phi^{*}(\mathbf{x}, \mathbf{y}) u(\mathbf{x}) d S+\int_{\Omega} \lambda^{\prime} u^{*}(\mathbf{x}, \mathbf{y}) u(\mathbf{x}) d \Omega+u(\mathbf{x})=0, \quad \mathbf{x} \in \Omega
$$

If $\mathbf{x}$ approaches the smooth boundary $(\mathbf{x} \in \partial \Omega)$, then the first term in (3.6) may be written as:

$$
\begin{gathered}
\lim \int_{\partial \Omega} \Phi^{*}(\mathbf{x}, \mathbf{y}) u(\mathbf{x}) d S=\int_{\partial \Omega}^{C P V} \Phi^{*}(\mathbf{x}, \mathbf{y}) u(\mathbf{x}) d S-\frac{1}{2} u(\mathbf{x}) \\
x \rightarrow \partial \Omega
\end{gathered}
$$

in the sense of a Cauchy Principal Value (CPV) integral.

For the understanding of the physical meaning of (3.7), we rewrite (3.3) and (3.6) as:

$$
\int_{\partial \Omega}^{C P V} \Phi^{*}(\mathbf{x}, \mathbf{y}) d S+\int_{\Omega} \lambda^{\prime} u^{*}(\mathbf{x}, \mathbf{y}) d \Omega+1 / 2=0, \quad x \in \partial \Omega
$$

and:

$$
\int_{\partial \Omega}^{C P V} \Phi^{*}(\mathbf{x}, \mathbf{y}) u(\mathbf{x}) d S+\int_{\Omega} \lambda^{\prime} u^{*}(\mathbf{x}, \mathbf{y}) u(\mathbf{x}) d \Omega+1 / 2 u(\mathbf{x})=0, \quad x \in \partial \Omega
$$

By (3.8) follows that only a half of the source function at point $\mathbf{x}$ is applied to the domain $\Omega$, when the point $\mathbf{x}$ approaches a smooth boundary, $\mathbf{x} \in \partial \Omega$.

Also, consider another weak form of eqn (2.5) by supposing the vector functions to be the gradients of an arbitrary function $u(\mathbf{y})$ in $\Omega$, chosen in such a way that they have constant values:

$$
u_{, k}(\mathbf{y})=u_{, k}(\mathbf{x}), \quad \text { for } \quad k=1,2,3
$$

Then the weak form of eqn (2.5) will be written as:

$$
\int_{\Omega}\left[u_{, i i}^{*}(\mathbf{x}, \mathbf{y})+\lambda^{\prime \prime} u^{*}(\mathbf{x}, \mathbf{y})\right] u_{, k}(\mathbf{y}) d \Omega+u_{, k}(\mathbf{x})=0
$$

By applying further the divergence theorem, then eqn (3.11) takes the form:

$$
\int_{\partial \Omega} \Phi^{*}(\mathbf{x}, \mathbf{y}) u_{, k}(\mathbf{x}) d S+\int_{\Omega} \lambda^{\prime} u^{*}(\mathbf{x}, \mathbf{y}) u_{, k}(\mathbf{x}) d \Omega+u_{, k}(\mathbf{x})=0
$$

Furthermore, the following property exists:

$$
\begin{aligned}
& \int_{\partial \Omega} n_{i}(\mathbf{y}) u_{, i}(\mathbf{x}) u_{, k}^{*}(\mathbf{x}, \mathbf{y}) d S-\int_{\partial \Omega} n_{k}(\mathbf{y}) u_{, i}(\mathbf{x}) u_{, i}^{*}(\mathbf{x}, \mathbf{y}) d S \\
& =\int_{\Omega} u_{i}(\mathbf{x}) u_{, k i}^{*}(\mathbf{x}, \mathbf{y}) d S-\int_{\partial \Omega} u_{, i}(\mathbf{x}) u_{, i k}^{*}(\mathbf{x}, \mathbf{y}) d S=0
\end{aligned}
$$

By adding eqs (3.12) and (3.13) then one obtains:

$$
\begin{array}{r}
\int_{\partial \Omega} n_{i}(\mathbf{y}) u_{, i}(\mathbf{x}) u_{, k}^{*}(\mathbf{x}, \mathbf{y}) d S-\int_{\partial \Omega} n_{k}(\mathbf{y}) u_{, i}(\mathbf{x}) u_{, i}^{*}(\mathbf{x}, \mathbf{y}) d S \\
+\int_{\partial \Omega} \Phi^{*}(\mathbf{x}, \mathbf{y}) u_{, k}^{*}(\mathbf{x}) d S+\int_{\Omega} \lambda^{\prime \prime} u^{*}(\mathbf{x}, \mathbf{y}) u_{, k}(\mathbf{x}) d \Omega+u_{, k}(\mathbf{x})=0
\end{array}
$$

which takes finally the form: 


$$
\begin{gathered}
\int_{\partial \Omega} n_{i}(\mathbf{y}) u_{, i}(\mathbf{x}) u_{, k}^{*}(\mathbf{x}, \mathbf{y}) d S+\int_{\partial \Omega} e_{i k t} R_{t} u(\mathbf{x}) u_{, i}^{*}(\mathbf{x}, \mathbf{y}) d S \\
+\int_{\partial \Omega} \lambda^{\prime} u^{*}(\mathbf{x}, \mathbf{y}) u_{, k}(\mathbf{x}) d \Omega+u_{, k}(\mathbf{x})=0
\end{gathered}
$$

\section{Analysis by Non-linear Singular Integral Equations}

Furthermore, the porous medium equation (2.1) will be written in another form, in order a singular integral equation representation to be applicable:

$$
\nabla^{2} p=-\nabla \ln \left(\frac{\lambda}{\Phi \xi_{c_{t}}}\right) \bullet \nabla p+\frac{\Phi \xi_{c_{t}}}{\lambda} \frac{\partial p}{\partial t}
$$

By applying further the Green Element Method, then eqn (4.1) reduces to the solution of a non-linear singular integral equation:

$$
\begin{gathered}
-\frac{\theta}{2 \pi} p\left(r_{i}\right)+\int_{\partial \Omega}\left(p \frac{\partial\left[\ln \left(r-r_{i}\right)\right]}{\partial n}-\ln \left(r-r_{i}\right) \frac{\partial p}{\partial n}\right) d S+ \\
+\iint_{\Omega} \ln \left(r-r_{i}\right)\left[-\nabla \ln \Lambda \bullet \nabla p+\frac{1}{\Lambda} \frac{\partial p}{\partial t}\right] d \Omega=0
\end{gathered}
$$

in which:

$$
\Lambda=\frac{\Phi \xi}{\lambda} c_{t}
$$

In order the non-linear singular integral equation (4.2) to be numerically evaluated, then the Singular Integral Operators Method (S.I.O.M.) will be used. Thus, the non-linear singular integral equation (4.2) is approximated by the formula:

$$
-\frac{\theta}{2 \pi} p\left(r_{i}\right)+\sum_{e=1}^{M}\left[\int_{\partial \Omega}\left(p \frac{\partial\left[\ln \left(r-r_{i}\right)\right]}{\partial n}-\ln \left(r-r_{i}\right) \frac{\partial p}{\partial n}\right) d S+\iint_{\Omega}\left(-\nabla \ln \Lambda \bullet \nabla p+\frac{1}{\Lambda} \frac{\partial p}{\partial t}\right) d \Omega\right]=0
$$

where $\mathrm{M}$ denotes the total number of elements.

Beyond the above, we introduce the following functions describing the pressure at any point in an element, in terms of the nodal pressures:

$$
p(x, y)=N_{j}(x, y) p_{j}
$$

By replacing (4.5) then eqn (4.4) takes the form:

$$
\sum_{e=1}^{M}\left(A_{i j}^{e} p_{j}+B_{i j}^{e} q_{j}-C_{i j l}^{e} \ln \Lambda_{j} p_{l}+D_{i j l}^{e} \frac{1}{\Lambda_{j}} \frac{\partial p_{l}}{\partial t}\right)=0, \quad i, j, l=1,2,3,4
$$

where:

$$
\begin{gathered}
A_{i j}^{e}=\int_{\partial \Omega} \frac{\partial\left[\ln \left(r-r_{i}\right)\right]}{\partial n} \Omega_{j} d S-\delta_{i j} \frac{\theta}{2 \pi} \\
B_{i j}^{e}=-\int_{\partial \Omega} \ln \left(r-r_{i}\right) \Omega_{j} d S \\
C_{i j l}^{e}=\iint_{\Omega_{j}} \ln \left(r-r_{i}\right)\left[\frac{\partial N_{j}}{\partial x} \frac{\partial N_{l}}{\partial x}+\frac{\partial N_{j}}{\partial y} \frac{\partial N_{l}}{\partial y}\right] d \Omega \\
D_{i j l}^{e}=\iint_{\Omega_{j}} \ln \left(r-r_{i}\right) N_{j} N_{l} d \Omega
\end{gathered}
$$




\section{Well Testings Applications in Heterogeneous Reservoirs}

The previous mentioned theory will be applied to the determination of a well testing, which will be checked in an heterogeneous reservoir with a permeability varying from $10 \mathrm{mD}$ to $300 \mathrm{mD}\left(1 \mathrm{mDarcy} \approx 10^{-12} \mathrm{~m}^{2}=1(\mu \mathrm{m})^{2}\right)$.

Hence, by using the Singular Integral Operators Method (S.I.O.M.) as described in the previous paragraphs, then it has been effected the computation of the pressure response from the well test conducted in the above heterogeneous reservoir. First of all the pressures were computed in variation with the time. Thus, Table 1 shows the pressure response with respect to the time.

Beyond the above, the pressure derivatives were computed with respect to the time, as shown in Table 2. Such derivatives are very much important of the well testings interpretation, as these are some distinct shapes and especially the characteristics of certain reservoir features.

The computational results of the pressures and the pressure derivatives are compared to the analytical solutions of the same well testing problem, if the reservoir was homogeneous with permeability equal to $50 \mathrm{mD}$. Thus, the analytical results are shown in Table 1 for the pressures and in Table 2 for the pressure derivatives, correspondingly. From the above Tables it can be seen that there is very small difference between the computational results and the analytical solutions for both the pressures and the pressure derivatives. On the other hand, the above mentioned small difference can be explained because of the diffusive nature of the pressure transport mechanism. Finally same results are shown, correspondingly in Figures 1 and 2, and in three-dimensional form in Figures 1a and 2a.

\section{Conclusions}

In the present investigation a mathematical model has been presented as an attempt to determine the properties of the reservoir materials. Thus, the study of the movement of oil reserves through porous media is very important for petroleum reservoir engineers. The above mentioned problem was reduced to the solution of a non-linear singular integral equation, which was numerically evaluated by using the Singular Integral Operators Method (S.I.O.M.).

Furthermore, several important properties of the porous medium equation, which is a Helmholtz differential equation, were analyzed and investigated. Thus, the fundamental solution of the porous medium equation was proposed and studied. Also, some basic properties of the fundamental solution were further investigated. These are very important in order the behavior of the non-linear singular integral equation to be well understood.

An application was finally given for a well testing to be checked when an heterogeneous oil reservoir is moving in a porous solid. The above problem was solved by using the Singular Integral Operators Method and thus the pressure response from the well test conducted in the above heterogeneous oil reservoir, was computed. Both the pressures and the pressure derivatives were computed and these values were compared to the analytical solutions of the same well testing problem, if the reservoir was homogeneous with a mean permeability.

Over the last years, non-linear singular integral equation methods have been used with a big success for the solution of several important engineering problems of structural analysis, elastodynamics, hydraulics, fluid mechanics and aerodynamics. For the numerical evaluation of the non-linear singular integral equations of the above problems, were used several aspects of the Singular Integral Operators Method (S.I.O.M.). Thus in the present research such methods were extended for the solution of oil reserves problems in petroleum reservoir engineering.

\section{References}

El Harrouni K., Ouazar D., Wrobel L. C., \& Cheng A. H. D. (1996). Global interpolation function based DRBEM applied to Darcy's flow in heterogeneous media. Engng Anal. Bound. Elem., 17, 281-285.

Kikani J. A., \& Horne R. N. (1992). Pressure-transient analysis of arbitrarily shaped reservoirs with the boundary element method. SPE Form. Eval., 53-60.

Koh L.S., \& Tiab D. (1993). A boundary element algorithm for modelling 3D horizontal wells problems using 2D grids. SPE Petrol. Computer Conf., New Orleans, LA, pp. 91-106.

Ladopoulos E. G. (1991). Non-linear integro-differential equations used in orthotropic spherical shell analysis. Mech. Res. Commun., 18, 111 - 119. http://dx.doi.org/10.1016/0093-6413(91)90038-X

Ladopoulos E. G. (1994). Non-linear integro-differential equations in sandwich plates stress analysis. Mech. Res. Commun., 21, 95 - 102. http://dx.doi.org/10.1016/0093-6413(94)90014-0 
Ladopoulos E. G. (1995). Non-linear singular integral computational analysis for unsteady flow problems. Renew. Energy, 6, 901 - 906. http://dx.doi.org/10.1016/0960-1481(95)00099-1

Ladopoulos E. G. (1995). Non-linear singular integral representation for unsteady inviscid flowfields of 2-D airfoils. Mech. Res. Commun., 22, 25 - 34. http://dx.doi.org/10.1016/0093-6413(94)00036-D

Ladopoulos E. G. (1997). Non-linear singular integral representation analysis for inviscid flowfields of unsteady airfoils. Int. J. Non-Lin. Mech., 32, 377 - 384. http://dx.doi.org/10.1023/A:1004246318082

Ladopoulos E. G. (2000). Non-linear multidimensional singular integral equations in 2-dimensional fluid mechanics analysis. Int. J. Non-Lin. Mech., 35, 701-708.

Ladopoulos E. G. (2000). Singular Integral Equations, Linear and Non-Linear Theory and its Applications in Science and Engineering. Springer Verlag, New York, Berlin.

Ladopoulos E. G. (2003). Non-linear two-dimensional aerodynamics by multidimensional singular integral computational analysis. Forsch. Ingen., 68, 105-110. http://dx.doi.org/10.1007/s10010-003-0114-7

Ladopoulos E. G. (2005). Non-linear singular integral equations in elastodynamics, by using Hilbert transformations. Nonlin. Anal., Real World Appl., 6, 531-536. http://dx.doi.org/10.1016/j.nonrwa.2004.10.002

Ladopoulos E. G., \& Zisis V. A. (2000). Non-linear finite-part singular integral equations arising in two-dimensional fluid mechanics. Nonlin. Anal., Th. Meth. Appl., 42, 277-290. http://dx.doi.org/10.1016/S0362-546X(98)00347-2

Lafe. O. E., \& Cheng A. H-D. (1987). A perturbation boundary element code for steady state groundwater flow in heterogeneous aquifers. Water Resour. Res., 23, 1079-1084.

Masukawa J., \& Horne R. N. (1988). Application of the boundary integral method to immiscible displacement problems. SPE Reserv. Engng. 1069-1077.

Numbere D. T., \& Tiab D. (1988). An improved streamline generating technique that uses the boundary (integral) element method. SPE Reserv. Engng., 1061-1068.

Onyejekwe O. O. (1997). A Green element treatment of isothermal flow with second order reaction. Int. Comm. Heat Mass Transfer, 24. 251-264. http://dx.doi.org/10.1016/S0735-1933(97)00011-0

Onyejekwe O. O. (1998). A boundary element - finite element equations solution to flow in heterogeneous porous media. Trans. Porous Media, 31, 293-312. http://dx.doi.org/10.1023/A:1006529122626

Onyejekwe O. O. (1998). Boundary integral procedures for unsaturated flow problems. Trans. Porous Media, 31, 313-330. http://dx.doi.org/10.1023/A:1006525124289

Sato K., \& Horne R. N. (1993). Perturbation boundary element method for heterogeneous reservoirs: Part 1 Steady - state flow problems. SPE Form. Eval., 306-314.

Sato K., \& Horne R. N. (1993). Perturbation boundary element method for heterogeneous reservoirs: Part 2 Transient flow problems. SPE Form. Eval., 315-322.

Taigbenu A. E., \& Onyejekwe O. O. (1997). Transient 1D transport equation simulated by a mixed Green element formulation. Int. J. Numer. Meth. Engng, 25, 437-454. http://dx.doi.org/10.1002/(SICI)1097-0363(19970830)25:4\%3C437::AID-FLD570\%3E3.0.CO;2-J 
Table 1.

\begin{tabular}{ccc}
\hline $\begin{array}{c}\text { Time } \\
\text { (hours) }\end{array}$ & $\begin{array}{c}\text { Pressure (psi) } \\
\text { S.I.O.M. }\end{array}$ & $\begin{array}{c}\text { Pressure (psi) } \\
\text { Analytical }\end{array}$ \\
\hline 0.002 & 7.003 & 7.022 \\
0.009 & 10.002 & 10.013 \\
0.015 & 12.002 & 12.031 \\
0.030 & 12.504 & 12.523 \\
0.040 & 13.003 & 13.014 \\
0.070 & 13.503 & 13.502 \\
0.100 & 14.002 & 14.033 \\
0.250 & 14.501 & 14.521 \\
0.400 & 15.004 & 15.032 \\
1.000 & 15.502 & 15.514 \\
2.000 & 16.004 & 16.023 \\
10.00 & 17.002 & 17.022 \\
30.00 & 17.504 & 17.524 \\
80.00 & 18.001 & 18.042 \\
100.00 & 19.003 & 19.032 \\
200.00 & 20.000 & 20.030 \\
400.00 & 20.000 & 20.020 \\
600.00 & 20.000 & 20.010 \\
1000.00 & 20.000 & 20.000 \\
\hline
\end{tabular}

Table 2.

\begin{tabular}{ccc}
\hline Time & Pressure Derivative (psi) & Pressure Derivative (psi) \\
(hours) & S.I.O.M. & 2.002 \\
0.002 & 1.504 & 2.003 \\
0.009 & 2.002 & 2.003 \\
0.015 & 2.001 & 2.002 \\
0.030 & 2.002 & 2.002 \\
0.040 & 2.003 & 2.003 \\
0.070 & 2.004 & 2.004 \\
0.100 & 2.002 & 2.002 \\
0.250 & 2.001 & 2.003 \\
0.400 & 2.003 & 2.002 \\
1.000 & 2.002 & 2.003 \\
2.000 & 2.004 & 2.002 \\
10.00 & 2.001 & 2.003 \\
30.00 & 2.003 & 2.003 \\
80.00 & 2.002 & 1.301 \\
100.00 & 1.001 & 0.800 \\
200.00 & 0.600 & 0.260 \\
400.00 & 0.250 & 0.060 \\
600.00 & 0.060 & 0.010 \\
1000.00 & 0.030 & \\
& & \\
\hline
\end{tabular}




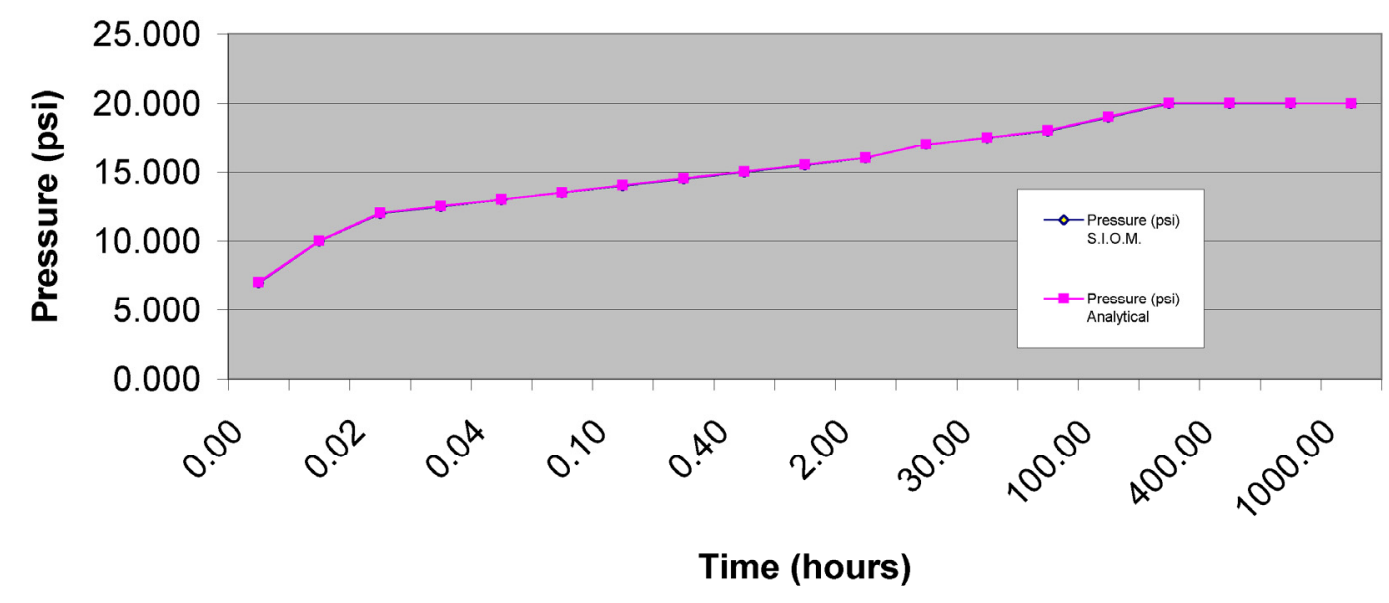

Figure 1. Pressure Response for Well Test in Heterogeneous Reservoir

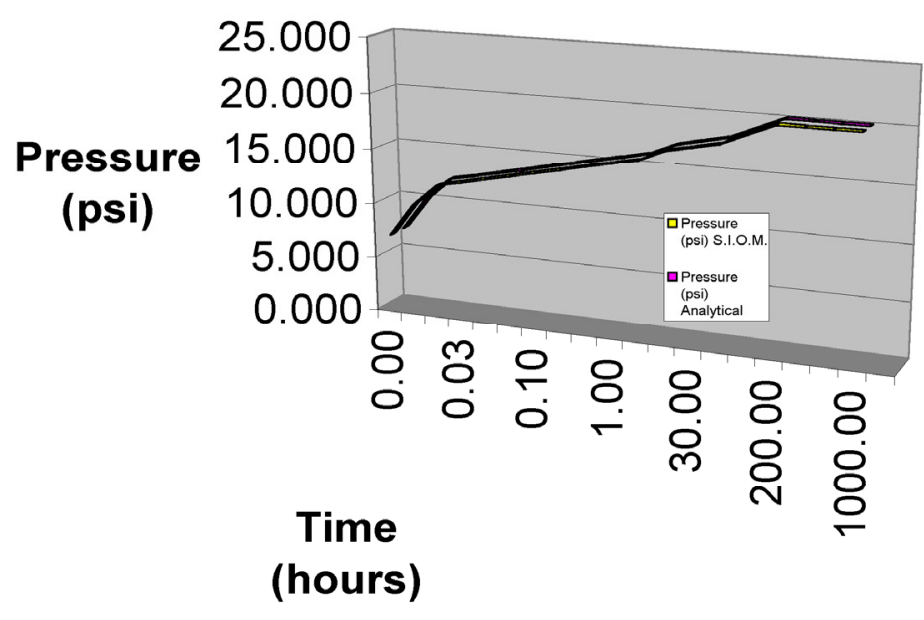

Figure 1a. 3-D Distribution of Pressure Response for Well Test in Heterogeneous Reservoir 


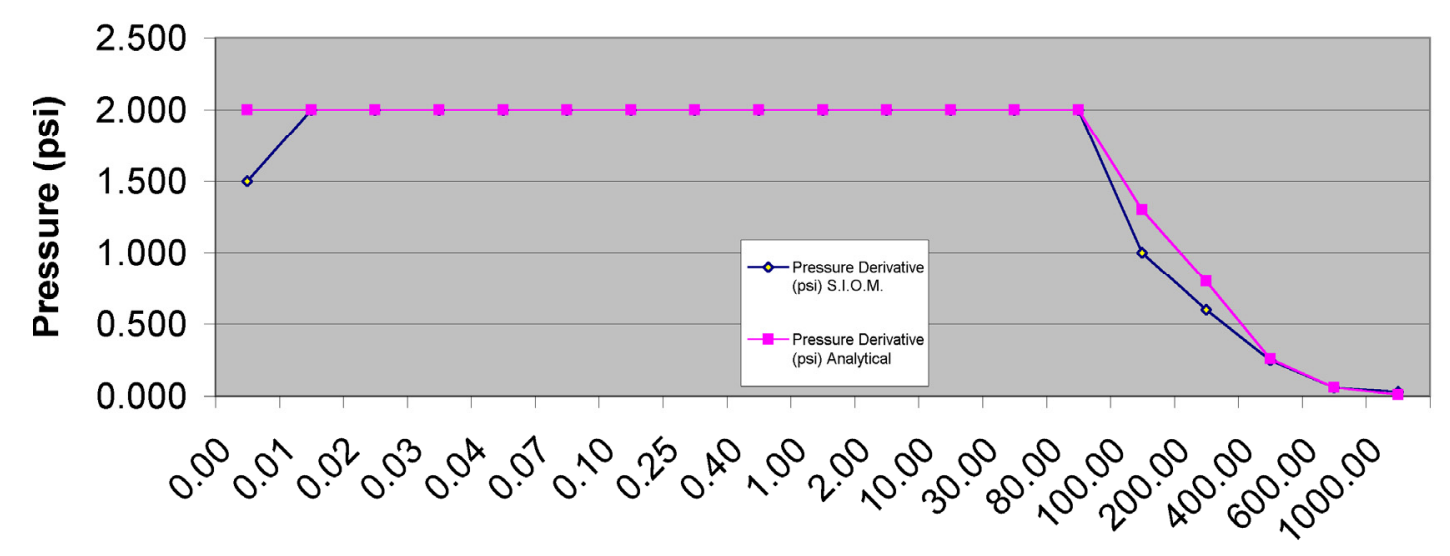

Time (hours)

Figure 2. Pressure Derivative for Well Test in Heterogeneous Reservoir

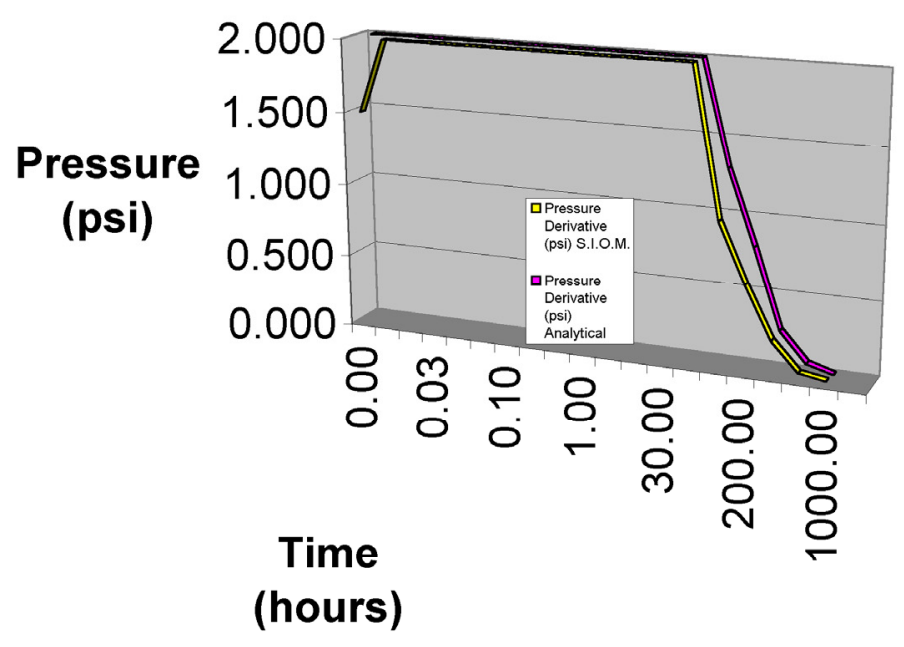

Figure 2a. 3-D Distribution of Pressure Derivative for Well Test in Heterogeneous Reservoir 